\title{
Paradox therapy for the treatment of social anxiety disorder: A case study
}

\author{
Ali Besharat $\mathrm{M}^{*}$ and Naghipoor M \\ Department of Psychology, University of Tehran, Tehran, Iran
}

\begin{abstract}
Social Anxiety Disorder (SAD) is a widespread debilitating and costly disorder for both the patient and the society. The high comorbidity rate of SAD with other psychological disorders as well as its impact upon increasing the burden of the disorder, call for more efficient approaches for its treatment. Evaluating the effectiveness of a new psychotherapeutic model for the treatment of SAD, shortly named PTC (Paradox + Timetable $=$ Cure), is introduced in this article. Three patients with SAD participated in a video recorded PTC therapy program. The results and the empirical evidence obtained from the treatment of the patients revealed a very positive complete outcome after three sessions for the first two patients and four sessions for the last one. The result of 24 to 36 months follow-up showed that the therapeutic changes were satisfactory, stable and permanent, during which no relapse was happened. Mechanisms of "paradoxical timetable", as the main PTC technique, and its adjustment to the treatment outcomes of the three patients are explained. It is concluded that the PTC psychotherapeutic model can be considered as a very shortterm, effective, efficient and yet economical approach for the treatment of SAD.
\end{abstract}

\section{Introduction}

Social anxiety disorder (SAD) is characterized by severe fear and anxiety in social situations or any other situations where the individual might be exposed to negative attention or judgment by others [1] SAD is a widespread, debilitating disorder which impairs important aspects of an individual's life such as financial and occupational stability, educational performance, mental health and the quality of life [2-4]. Apart from having multifarious damaging and incapacitating consequences for patients and their family, SAD is considered a costly disorder for the whole society as well. Research shows that the direct costs of SAD, such as medication and psychotherapy, are as high as its indirect costs including productivity loss [5,6]. Taking into account these clinical facts, it is necessary to offer a timely and efficient treatment for SAD. The high comorbidity rate of SAD with other psychological disorders [3,7] and its impact upon increasing the burden of the disorder [8] accentuates the necessity of treating this disorder. The present paper offers a paradoxical therapeutic (PT) model for treating SAD. This paradoxical psychotherapeutic model, shortly named PTC [9], is a method of treating psychological disorders that has been able to resolve many of the limitations and shortcomings of other existing treatments.

\section{A brief summary of PTC- treatment protocol}

PTC has its theoretical roots in psychodynamic, psychoanalytic and systemic theories. In terms of practice and therapeutic techniques, however, it is practically loyal to behavioral techniques [9]. PTC consists of two main techniques, paradox and timetable. According to this joint technique, which consists of a paradox and a timetable, the exact symptoms are prescribed to the patient. The patient has to recreate and re-experience the symptoms according to the instructions given by the therapist on certain times during the day within a specific time span. In PTC, the two techniques are always assigned together according to the inseparability principle. In sum, the combination of the two techniques, the inseparability principle that links paradox and timetable, and the prescription of the exact symptom are the main characteristics of PTC.

Two more points need to be explained to the patient. Firstly, the patient has to start doing the task from at least a day after the ongoing session and cannot start on the same day. Secondly - and this is more important - the therapist must ask the patient to not look for any other treatment nor change in any way, but to simply stick to the task. The therapist must explain to the patient that although they must do the task on certain times during the day, this does not mean that they are free to fight their symptoms or disorder at other times. The patient must be told that he/she is not allowed to do anything on his/her own to cure the disorder before the treatment is over. Quite the contrary, the symptoms must be allowed to come and go freely until the treatment is completed. The delayed initiation of the tasks along with refraining from fighting the symptoms and problematic behaviors are other characteristics of the PTC model.

The first session ends, and the second session is set when the tasks are assigned, and the patient understands exactly what to do and what not to do. The interval between sessions in PTC is usually two weeks. In the interval, the patient experiences the lack of anxiety attacks. Certain patients even take the opportunity to expose themselves to social situations in order to make sure that they no longer become anxious. As time goes by and the sessions are held, the therapist can further increase the two-week interval between the sessions if he/she decides that the pace of the treatment is in line with the PTC model.

${ }^{\star}$ Correspondence to: Mohammad Ali Besharat, Department of Psychology, University of Tehran, Tehran, Iran, E-mail: besharat@ut.ac.ir

Key words: psychotherapy, paradox, timetable, PTC, SAD

Received: October 15, 2019; Accepted: November 04, 2019; Published: November 06, 2019 
At the beginning of each session, the therapist receives a complete account from the patient on the tasks, the changes and the probable obstacles. The therapist examines the account attentively and bases and prescribes the subsequent tasks on it. The tasks that a patient must carry out in the PTC psychotherapeutic model are usually decremental (another characteristic of the PTC therapeutic model). PTC is a shortterm therapeutic method, and the number of sessions is not determined beforehand, but changes from patient to patient. A patient might be cured after two sessions while another may need about five. The therapist intervenes and manages the treatment as long as necessary. The patient's condition determines when the treatment will come to an end. This happens when the patient no longer suffers from the problem or the disorder for which he/she had took therapy, when he/she feels that he/she is no longer a patient, when he/she has the ability to manage the conditions that intensify the symptoms of the disorder and, finally, when the disorder is cured. The patient's experience directly determines the end of the treatment.

\section{Method and case presentation}

Three patients suffering from SAD participated in the present study at an academic clinic center. They signed an informed consent document prior to performing the treatment sessions for both treatment and use of the anonymized case information for educational, learning, and research purposes. A written consent for video recording of all treatment sessions was also signed by the patients. For initial evaluation the patients participated in a diagnostic interview [1], the Liebowitz Social Anxiety Scale-Self-Report [LSAS-SR]; [10,11], the Beck Anxiety Inventory [BAI; 12], the Beck Depression InventoryII [BDI-II; 13], and the Treatment Outcome Subjective Rating Scale [TOSRS; 14]. The patients met criteria for DSM-5 SAD, as well as a total score of 79.5, 76.2, and 81.7 for the LSAS-SR, respectively. A total score of 41.7, 37.9, 43.5 for BAI; and a total score of 9.7, 23.9, and 13.7 for BDI-II, respectively. Subjective ratings on SAD on a scale of 0 (none) to 100 (extreme) percent were obtained at regular intervals. Social anxiety symptoms during the first session was 100 out of 100 for the patients 1 and 3 , and 90 out of 100 for the patient 2 . At their last session, SAD symptoms reduced to $10 / 100$ for the first patient, 25/100 for the second patient, and 5/100 for the third patient (Figures 1-3). At post treatment, total scores of the LSAS-SR, BAI, and BDI-II reduced to 14.1, 10.7, and 6.3 for the first patient: 15.7, 14.3, and 11.9 for the second patient; 13.9, 6.3, and 9.1 for the third patient, respectively. The following is the session by session accounts of the PTC therapeutic model as applied to three patients diagnosed with SAD.

\section{The application of PTC to SAD}

Case 1: Miss. MK 32 years old, single, referred to an academic clinic centre complaining about severe anxiety for which she had been receiving medication for 12 more than years. Based on DSM-5 [1], it was determined in the first session that the patient had the criteria for social anxiety disorder, including severe panic attacks in social situations and interactions and during exams. The main symptoms of the disorder included: rapid heart rate, extreme fear of a supposedly bad event happening, shaky voice, severe pain and spasm of muscles in the neck, shoulders and hands. The patient has had the anxiety symptoms since her childhood, however they worsened when she reached 13 and have intensified ever since. They peaked when she reached 20 and she had to take medication to alleviate them. The daily medication used by the patient includes Nortriptyline and Inderal $10 \mathrm{ml}$. Her family history shows that her mother and her two elder sisters had anxiety disorders too. At the end of the first session, the following assignments

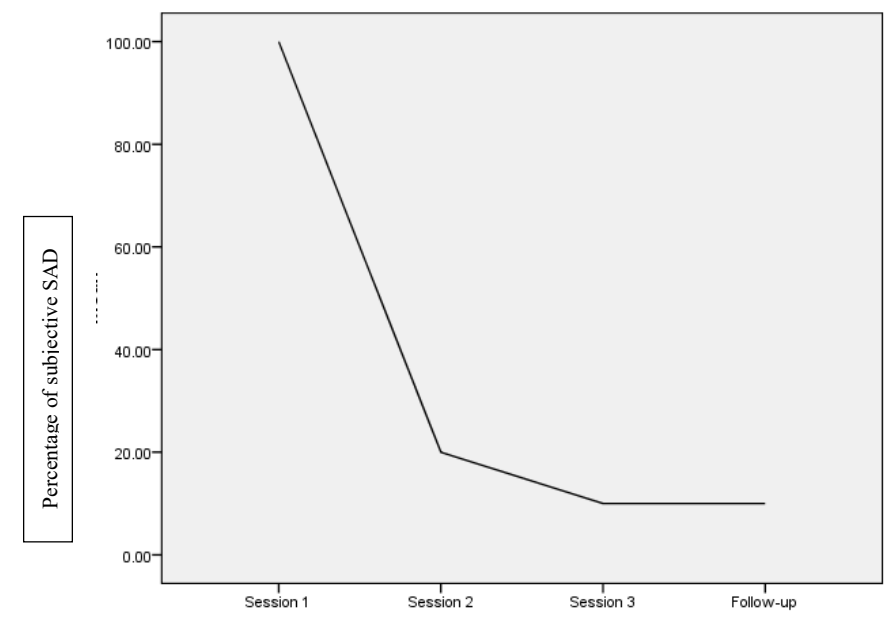

Figure 1. Subjective ratings of SAD by session

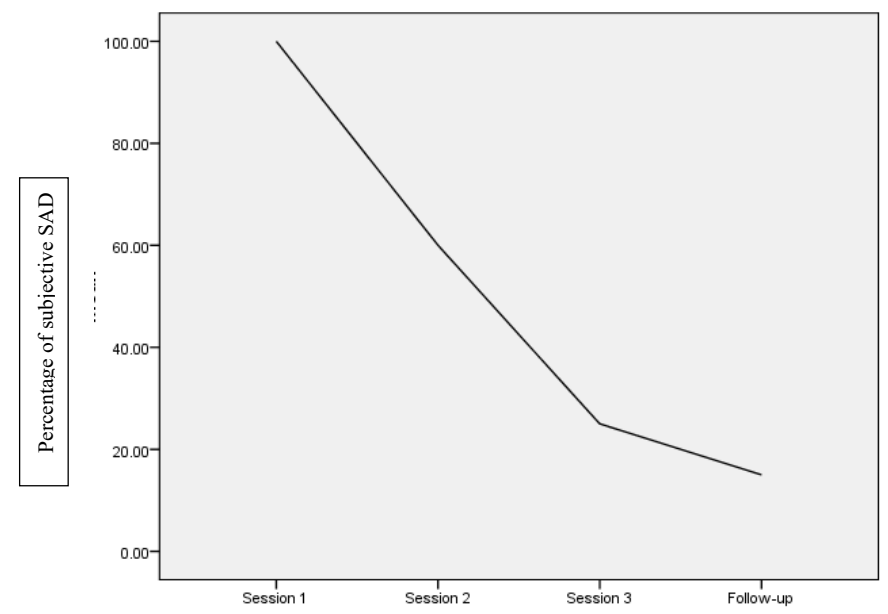

Figure 2. Subjective ratings of SAD by session

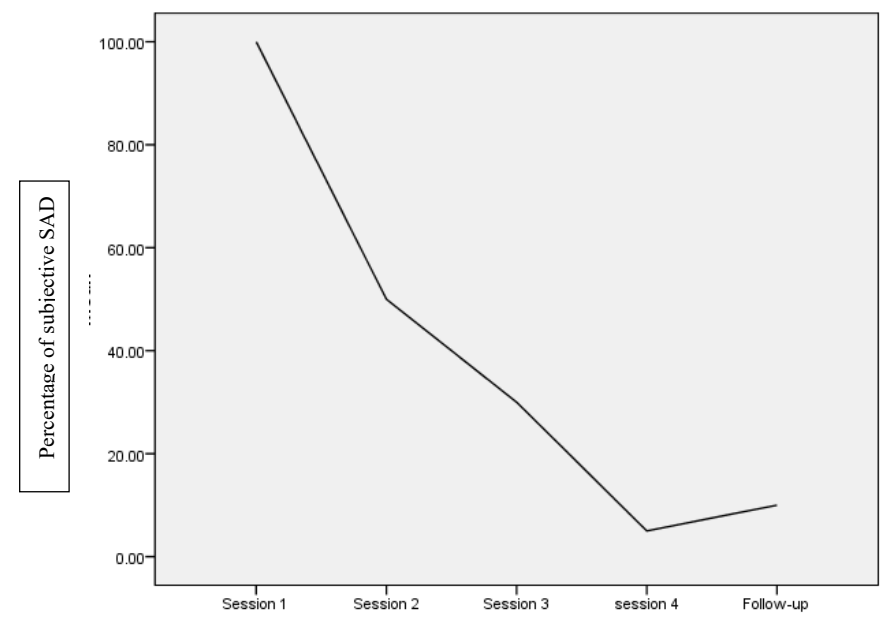

Figure 3. Subjective ratings of SAD by session

related to the paradoxical timetable were given to the patient: for the first week, recreating the anxieties, fears and concerns three times a day (at 10:00, 17:00, and 22:00), each time for 15 minutes at the maximum (the therapist had prescribed 10 minutes for each time, but due to the patient's request, he agreed to increase the time to 15 
minutes); and for the second week, three times a day (at 10:00, 17:00, and 22:00), each time for 10 minutes at the maximum. After a twoweek interval, the patient attended the second session with a cheerful expression and reported that the exercises had been really helpful. $\mathrm{He}$ had felt no anxiety or stress for the last two weeks and did not need to take medication. "Before this, if I didn't take drugs for two days I couldn't sleep at all on the third day. But, although I didn't take any drugs during the last two weeks, I felt good and I was able to sleep well." The patient added that for the last two weeks, she was able to attend a professional interview successfully. With regard to the patient's progress, it was decided that she should do the exercises twice a day (at 10:00 and 22:00), each time for 5 minutes. The third session was held after another two-week interval. Having ascertained that the treatment was complete, and the symptoms of the social anxiety disorder had disappeared, and with the patient's agreement, the therapist concluded the therapy. It was explained to the patient that if the symptoms of the disorder reappear, she must wait for 5 to 7 days before assigning similar exercises for herself and carry them out to the extent that the problem is solved. In line with the PTC model, the therapist taught the patient how to be her own therapist. Nonetheless, he assured her that she can have therapy anytime she wants.

Case 2: Miss. RA 33 years old, single, visited an academic clinic centre complaining about social anxiety, relatively low mood and shaky self-confidence. The symptoms of the disorder have existed on since her childhood and adolescence. However, they have become more severe, annoying and dominant for two years ago and have disrupted her personal and professional life. Her symptoms fulfilled the DSM-5 [1] criteria for social anxiety disorder including severe stress at social situations among colleagues, fear of being addressed by others and avoidance behaviors. The patient's social anxiety is so much that she has difficulty going to a shop and buying things: "Even while buying a bottle of milk, or when using my credit card to pay for things, I feel so stressed that my hands shake noticeably." Among her family members, her mother suffers from anxiety disorder, too. At the end of the first session, as is the case with the PTC model, the following paradoxical timetable was arranged for the patient so that she can recreate the symptoms of the disorder and re-experience them voluntarily through predetermined occasions: three times a day for the first week (at 10:30, 17:00, and 20:00), each time for 10 minutes; twice a day for the second week (10:30 and 17:00), each time for 10 minutes at the maximum. The patient's report at the beginning of the second session showed that she had been able to complete the exercises during the first week, but she failed to do them during the second week due to some trip that had suddenly come up. However, she said that she felt better in general. Later on, during the session, the patient said that in case the relationship (with the opposite sex) turns into a romantic or emotional one, her anxiety worsens. Having inquired about her emotional as well as family relationships, the therapist asked her to do the paradoxical timetable exercises three times a day (at 10:30, 17:00, and 20:00), each time for 10 minutes at the maximum. The patient was told that this time, the content of her exercises must be related to her emotional relationships. At the beginning of the third session, the patient who looked happy and contented, reported that she had carried out the exercises and had gotten much better: "After doing the exercises for three days, I had to get ready for a professional appointment which had romantic significance for me as well. It was perfect. I didn't feel any stress, either before or throughout the appointment. And I felt great after it, and I didn't feel the need to do the exercises for several days." Taking into account the patient's satisfactory condition, the therapist asked her to continue doing the paradoxical timetable exercises a little bit more, once a day (at 10:30) for 5 minutes.

Case 3: Mr. JM 25 years old, single, referred to an academic clinic centre complaining about SAD as well as specific phobias. Based on DSM-5 [1], it was determined in the first session that the patient had the criteria for social anxiety disorder. He explained his problem thus during the first session: when I was a child, my heart started racing madly whenever I was asked questions in class. Blood rushed to my neck and face, my hands started sweating and I couldn't breathe. If I had to stand up, my knees would shake. Gradually, it got even worse. Now, even when I'm among friends and they introduce me to somebody new, I feel anxious. I feel the same way at work and at parties. I spend so much energy to control myself that I no longer enjoy being among other people. Two years ago, I suffered from depression for which I took drugs. I also received CBT which did not help. My friends and social relationships have decreased because of my problem. Later on during the session, the patient explained that during childhood, he had seriously suffered due to family problems that had left him with some disturbing memories of domestic violence (some of which had been directed at him). At the time of visiting the therapist, the patient took no drugs. At the end of the first session, the following exercises were assigned for the patient: recreating anxieties, emotional and somatic symptoms through visualizing the disturbing past experiences 3 times a day (at 9:00, 14:00, and 23:00) for two weeks, each time for 10 minutes. At the beginning of the second session, the patient reported that he had been able to complete most of the exercises and "generally, some of the situations that made me anxious before, didn't trouble me anymore, and the anxiety attached to some other situations decreased significantly. On the third or the fourth day, when I found myself in a situation that could make me really anxious before, I felt nothing. Everything was fine, except for two occasions. One was when I was in class and I felt stressed, and the other one was when I went to the stadium to watch a soccer match." The therapist prescribed new exercises for the patient according to his report and gave him the following explanation: in order to move from the visualization to actuality, to get ready to experience real social situations such as the class, you need to visualize the worst case scenario in that situation. Do this twice a day (at 9:00 and 23:00), each time for 10 minutes at the maximum. The patient's report in the third session showed that he had improved further and the symptoms of the disorder had decreased by 70 percent. Taking into account the history of physical violence and severe family conflicts, especially between the patient and his mother, it was decided that he should recreate and reexperience those disturbing memories three times a day (at 9:00, 15:00, and 23;00), each time for 10 minutes at the maximum. The patient was asked to do the new exercise on all the three occasions every day for the first week, and, for the second week, to do the anxiety exercise on the first session (9:00) and allocate the other two sessions (15:00 and 23:00) to the exercises related to her mother. On the fourth session, the patient reported that all the anxiety symptoms had disappeared, and he was able to present a class conference without any anxiety. Doing the exercises related to his mother had enabled him to get rid of his anger and hatred for his mother: "No matter how hard I tried, I couldn't feel that strong anger and hatred. Before this, I couldn't forgive her at all. But now, I am totally ready to forgive her." I went to see her last week, and it was great, and I feel much closer to her now." When asked about the symptoms of depression, the patient added that he had felt much better since the beginning of therapy and had not experienced a low mood. The therapist asked the patient to do the exercises for four more weeks, once a day (at 23:00), for five minutes. "You are to do the exercises related to your mother and the anxiety exercises on alternate 
days. You must cease doing the exercises after four weeks. I believe that you will feel no anxiety, depression or problem after this period. But, if you feel after six months, or a year, that the anxieties and conflicts are coming back, first wait for about a week. If they do not disappear through this time, you can arrange for yourself exercises similar to the one we arranged together. The result will be the same and you will no longer need to come to therapy. But if you feel depressed, arrange a session and we will talk about it."

\section{Evaluating the treatment outcomes and follow-up}

Evaluation of the treatment, which was based on the patients' answers to a question on a scale from 0 to 100 as well as an openended question, showed $90 \%, 75 \%$, and $95 \%$ recovery for the patients at the end of therapy, respectively. A 36-, 30- and 24-month followup evaluation revealed that the improvements gained by PTC have continued for all the three patients at $90 \%$ for the first patient, $85 \%$ for the second patient, and $90 \%$ for the third patient (Figures 1-3).

\section{Discussion}

How can the paradoxical timetable bring about immediate, deep clinical change that is stable for a long time? What changes in the patient through this type of intervention? Has the treatment been focused merely on decreasing and controlling the symptoms, as can be seen in traditional cognitive-behavioral approaches? [15] Have they been centered on tolerating the symptoms, as in inhibitory learning approaches [16]? Or have they been oriented toward the ability to accept (cope with) the symptoms, in line with acceptance and commitment therapy $[17,18]$ ? How and in what level have such comprehensive therapeutic changes been brought about so fast? How are the therapeutic changes in the PTC model so stable? In the following, we answer these questions explaining the mechanisms through which the paradoxical timetable works.

\section{Effective mechanisms of paradoxical timetable}

There are four main mechanisms in the PTC model that can determine therapeutic changes: 1) ordering-artificializing; 2) Breaking the link between the symptom and the anxiety; 3 ) changing the meaning of the symptom; 4) ego strength. The therapist and the patient agree on a paradoxical timetable which consists of specific, regular sessions. The therapist asks the patient to recreate and re-experience of his/her own accord the symptoms of social anxiety that appear in social and practical situations and make the patient anxious, panicked and desperate. The patient must, on the one hand, recreate the symptoms that appear in his/her mind involuntarily and out of his/her control and make him/ her suffer, according to the instruction of the therapist (ordering). On the other hand, the patient must re-experience real symptoms and behaviors through artificially acting them out (artificializing). This mechanism, along with the principles of prescribing the exact symptom and the delayed beginning of the paradoxical tasks of the timetable, can either decrease or remove the anxiety associated with doing the tasks, and increases the chance for the artificial recreation of the symptoms. The accounts of all the three patients showed that they were able to follow the instructions given by the therapist and carry out the tasks assigned without any problem. As a result, the link between the symptom and the anxiety is broken this is the second mechanism.

Anxiety, fear and negative emotions are at the heart of psychological disorders, including the social anxiety disorder. The disorder and its symptoms are not considered pathologic without such anxiety. When the relationship between symptoms and anxiety is broken, the symptom is no longer enforced or obligatory and cannot annoy the individual. The individual has control over it and can remove it from his behavior and life any time he wants. In the PTC model, the patient is not asked to control and manage the symptoms, neither is he/she asked to tolerate or accept them. The patient is asked to live as before, the only difference being that he/she must do certain tasks according to a certain timetable. Repeating the tasks in the interval between the two sessions gives the patient the opportunity to examine this hypothesis and believe in its existence. Such practical emotional experience can easily change the meaning of the symptom.

Carrying out the paradoxical symptoms breaks the link between the symptom and the anxiety and changes the patient's cognition and the perceived meaning of the symptoms and the disorder. When the patient realizes through personal experience that the symptoms are no longer pathological, he/she is able to change the meaning and value of those symptoms. Repeating the tasks gives the patient the chance to examine and re-experience the futility of the symptoms. As a result of such experience, the patient's previous beliefs concerning the symptoms changes. The PTC psychotherapeutic model does not resort to common instructive or cognitive approaches for changing the meaning of the symptom. Part of the anxiety experience by the patient arises from the negative meaning that the disorder has both for the patient and for the system in which he/she lives. This meaning changes through prescribing the exact symptom in the first session; a positive change that decreases the anxiety associated with the symptom, removing the negative meaning of the symptom, and making it easier for the patient to carry out the tasks. Changing the meaning of the symptom occurs first through removing the anxiety associated with the symptoms and then through carrying out the paradoxical tasks. In the next step, the patient's actual experience in doing the paradoxical tasks aims at changing the meaning of the symptom. Such curing experience, from artificializing-ordering to breaking the link between the symptom and the anxiety as well as changing the symptom's meaning, all contribute to ego strength.

Ego strength and authority is the ultimate goal in the PTC model. Under a psychological disorder, this authority is broken and weakened. In other words, a psychological problem is the direct result of the weakness on the part of the ego to manage psychological conflicts and environmental demands. These conflicts comprise the reality of the mental structure and the outer world from the cradle to the grave. Such conflicts and the stress and anxiety associated with them are prerequisites of a standard life and cannot be problematic in case the ego is strong enough. Psychological problems and disorders occur when the ego is weak and impotent while encountering such conflicts and fails to manage them. That is why the ultimate goal of the PTC model is to strengthen the ego. In case the ego is weak, the conflicts that are mainly psychological are continued, intensified, and upset the psychological order through one form of disorder [19-21].

In PTC, the strengthening of the ego starts from the first session after removing the anxiety associated with the symptoms which happens through prescribing the exact symptoms of the disorder. Carrying out the paradoxical tasks creates a free of anxiety atmosphere for the patient and enables him/her to fight the disorder. The patient is guaranteed to win this fight against the disorder. Patient's practical emotional experience which occurs through the paradoxical tasks between the first and the second session, tilts the pathological imbalance in favor of the greater authority for the ego. This genuine emotional experience, which is at the heart of all the psychodynamic approaches and the PTC model as well, increases the ego authority and strength either suddenly or after the experience has been repeated several times. When the ego 
is strengthened, anxiety and disorder are resolved, and the treatment is completed. The accounts and comments given by the patients proved that the ego had truly been strengthened.

\section{Conclusion}

The results of the PTC model for the three patients showed that the PTC model is a successful, very short-term approach that can be used to treat social anxiety disorder. The instruction given by the therapist as well as the tasks assigned are all defined through principles that aim to decrease to the minimum the anxiety associated with the task and increase the probability of the patient's compliance to the instructions. The inseparability principle between the paradox and the timetable, the principle of prescribing the exact symptom, the principle of delayed beginning of the tasks and the exclusive reliance on the practical treatment techniques prepare the ground for the recreation of an emotional experience with the utmost rapidity and has the treatment objectives realized. Ego strength is the ultimate goal of the PTC model. Increasing ego strength and authority increases the stability of treatment changes and decreases greatly the risk for relapse. The PTC model is a very short-term approach with immediate results which is easy to carry out for both the therapist and the patient. The simple nature of the PTC model and its techniques is one of the major advantages of this model. This simplicity enables the patient to turn into a therapist by the end of the treatment process. For instance, in answering the first patient's question about whether the disorder might relapse or not, the therapist says: if the disorder appears again, wait for 5 to 7 days in order to make sure it has relapsed. Then, you can do one of two things: either visit the therapist again, or make a timetable for yourself and stick to it. All you need is to prescribe for yourself the task I assigned to you and carry it out. I suggest that you serve as your own therapist in case the disorder reappears.

\section{Conflict of interest}

The authors declare that they have no conflict of interest.

\section{Ethical approval}

All procedures performed in studies involving human participants were in accordance with the ethical standards of the institutional and/ or national research committee and with the 1964 Helsinki declaration and its later amendments or comparable ethical standards.

\section{Informed consent}

Informed consent was obtained from all individual participants included in the study.

\section{Funding and acknowledgement}

The authors would like to acknowledge the financial support of University of Tehran for this research under grant number $5106003 / 1 / 87$.

\section{References}

1. American Psychiatric Association (2013) Diagnostic and statistical manual of mental disorders (5th edn) Arlington, VA: American Psychiatric Publishing.

2. Fink M, Akimova E, Spindelegger C, Hahn A, Lanzenberger R, et al. (2009) Social anxiety disorder: Epidemiology, biology, and treatment. Psychiatr Danub 21: 533-542.

3. Kessler RC, Avenevoli S, McLaughlin KA, Green JG, Lakoma MD, et al. (2012) Lifetime co-morbidity of DSM-IV disorders in the US national comorbidity survey replication adolescent supplement (NCS-A). Psychol Med 42: 1997-2010. [Crossref]

4. Szafranski DD, Talkovsky AM, Farris SG, Norton, PJ (2014) Comorbidity: Social anxiety disorder and psychiatric comorbidity are not shy to co-occur. In J. W. Weeks (Ed.), The Wiley-Blackwell handbook of social anxiety disorder. John Wiley and Sons, Ltd, Chichester, UK, pp: 201-222.

5. Acarturk C, Cuijpers P, van Straten A, de Graaf R (2009) Psychological treatment of social anxiety disorder: A meta-analysis. Psychol Med 39: 241-254.

6. Smit F, Cuijpers P, Oostenbrink J, Batelaan N, de Graaf R, et al. (2006) Costs of nine common mental disorders: implications for curative and preventive psychiatry. J Ment Health Policy Econ 9: 193-200. [Crossref]

7. Johnson SU, Hoffart A, Nordahl HM, Wampold BE (2017) Metacognitive therapy versus disorder-specific CBT for comorbid anxiety disorders: A randomized controlled trial. J Anxiety Disord 50: 103-112. [Crossref]

8. Gadermann AM, Alonso J, Vilagut G, Zaslavsky AM, Kessler RC (2012) Comorbidity and disease burden in the National Comorbidity Survey Replication (NCS-R). Depres Anxiety 29: 797-806. [Crossref]

9. Besharat MA (2017) Paradox + Timetable $=$ Cure (PTC): Perfect model of treatment for psychological disorders (A practical guide). Roshd Press, Tehran. [Farsi]

10. Fresco DM, Coles ME, Heimberg RG, Liebowitz MR, Hami S, et al. (2001) The Liebowitz Social Anxiety Scale: A comparison of the psychometric properties of selfreport and clinician-administered formats. Psychol Med 31: 1025-1035.

11. Liebowitz MR (1987) Social phobia. Mod Probl Pharmacopsychiatry 22: 141-173. [Crossref]

12. Beck AT, Epstein N, Brown G, Steer RA (1988) An inventory for measuring clinical anxiety: Psychometric properties. J Consult Clin Psychol 56: 893-897.

13. Beck AT, Steer RA, Garbin MG (1988) Psychometric properties of the Beck Depression Inventory: twenty-five years of evaluation. Clin Psychol Rev 8: 77-100.

14. Besharat MA (2004) Treatment Outcome Subjective Rating Scale. Unpublished research report. University of Tehran, Tehran. [Farsi]

15. Craske MG, Barlow DH (2007) Mastery of your anxiety and panic: Therapist guide (4th edn). Oxford University Press, New York.

16. Craske MG, Kircanski K, Zelikowsky M, Mystkowski J, Chowdhury N, et al. (2008) Optimizing inhibitory learning during exposure therapy. Behav Res Ther 46: 5-27. [Crossref]

17. Eifert GH, Forsyth JP (2005) Acceptance and commitment therapy for anxiety disorders: A practitioner's treatment guide to using mindfulness, acceptance, and values-based behaviour change strategies. Guilford Press, New York.

18. Hayes SC, Strosahl KD, Wilson KG (1999) Acceptance and commitment therapy: An experiential approach to behavior change. Guilford Press, New York.

19. Besharat MA (2018) Paradox + Timetable $=$ Cure (PTC): Perfect model of therapy with couples (A practical guide). Roshd Press, Tehran. [Farsi].

20. Besharat MA (2019) Theoretical foundations of PTC. Roshd Press, Tehran. [Farsi- in press].

21. Besharat MA, Naghipoor M (2019) The application of a new model of paradox therapy for the treatment of illness anxiety disorder: A case report. Clin Case Rep Int 3: 1-3.

Copyright: (C2019 Ali Besharat M. This is an open-access article distributed under the terms of the Creative Commons Attribution License, which permits unrestricted use, distribution, and reproduction in any medium, provided the original author and source are credited. 